\title{
Visual perceptual load induces inattentional deafness
}

\author{
James S. P. Macdonald • Nilli Lavie
}

Published online: 25 May 2011

(C) The Author(s) 2011. This article is published with open access at Springerlink.com

\begin{abstract}
In this article, we establish a new phenomenon of "inattentional deafness" and highlight the level of load on visual attention as a critical determinant of this phenomenon. In three experiments, we modified an inattentional blindness paradigm to assess inattentional deafness. Participants made either a low- or high-load visual discrimination concerning a cross shape (respectively, a discrimination of line color or of line length with a subtle length difference). A brief pure tone was presented simultaneously with the visual task display on a final trial. Failures to notice the presence of this tone (i.e., inattentional deafness) reached a rate of $79 \%$ in the high-visual-load condition, significantly more than in the low-load condition. These findings establish the phenomenon of inattentional deafness under visual load, thereby extending the load theory of attention (e.g., Lavie, Journal of Experimental Psychology. Human Perception and Performance, 25, 596-616, 1995) to address the cross-modal effects of visual perceptual load.
\end{abstract}

Keywords Attention · Load - Inattentional blindness .

Cross-modal $\cdot$ Awareness

A wealth of research has suggested that the extent to which focused attention on a task results in reduced perception of irrelevant information depends on the level of perceptual load in the task (see Lavie, 2005, 2010, for reviews). The

J. S. P. Macdonald

Department of Experimental Psychology, University of Oxford, Oxford, UK

N. Lavie $(\bowtie)$

Institute of Cognitive Neuroscience, University College London, 17 Queen Square,

London WC1N 3AR, UK

e-mail: n.lavie@ucl.ac.uk concept of perceptual load corresponds to the amount of information involved in the perceptual processing of the task stimuli. This is operationally defined in terms of either the number of different task stimuli or the perceptual requirements of the task performed on the same stimuli (e.g., a simple feature detection task involves less perceptual load than a complex perceptual discrimination task). Many studies have found that low-load tasks leave spare capacity that spills over to the processing of taskirrelevant information. In contrast, tasks involving higher perceptual load consume all or most of attentional capacity, leaving little or none remaining for processing any task-irrelevant information (Lavie, 1995; Lavie \& Tsal, 1994). Indeed, reduced perceptual processing of taskirrelevant information in high-load tasks leads to various forms of "inattentional blindness" (Mack \& Rock, 1998). These range from failures to recognize meaningful distractor objects (Jenkins, Lavie, \& Driver, 2005; Lavie, Lin, Zokaei, \& Thoma, 2009) to failures to notice the mere presence of stimuli (Carmel, Saker, Rees, \& Lavie, 2007; Cartwright-Finch \& Lavie, 2007; Macdonald \& Lavie, 2008; Neisser \& Becklen, 1975; Simons \& Chabris, 1999).

To date, the majority of evidence for the effects of perceptual load on awareness, and conversely on inattentional blindness, has been obtained within the visual modality. The world, however, is multisensory: We are constantly bombarded by information from all sensory modalities simultaneously. In everyday life, we find ourselves focusing attention on a task in one modalityfor example, reading - while also being exposed to information from other modalities - such as people talking nearby. The question of whether perception of the irrelevant auditory information depends on the level of perceptual load in the visual task is important for understanding the effects of attention on perception, in a way that is more 
applicable to the functioning of attention in our multisensory world. Furthermore, whether or not an auditory stimulus reaches awareness while attention is engaged in a visual task of high perceptual load is of particular importance, since there are many situations in everyday life in which such "inattentional deafness" would be highly undesirable. For example, would the sound of your phone ringing, or of your car alarm somewhere down the street, be noticed when you were engaged in a computer task involving high visual processing load? Would a car horn be noticed when you were attending to a visually loaded billboard?

Hence, we ask whether perceptual load in a visual attention task will modulate conscious awareness of taskunrelated auditory tones. For this purpose, we modified an inattentional blindness paradigm (e.g., Cartwright-Finch \& Lavie, 2007) to investigate the effects of visual task demands on the incidence of inattentional deafness. Akin to the study of Cartwright-Finch and Lavie, participants performed a visual discrimination task of either low or high perceptual load for a few trials. In a change from Cartwright-Finch and Lavie's design, however, in the last experimental trial a tone was presented, and participants were asked whether they had noticed the tone.

Opposing predictions of the results can be derived from previous suggestions that perceptual capacity is either modality specific (Allport, Antonis, \& Reynolds, 1972; Duncan, Martens, \& Ward, 1997; McLeod, 1977; Treisman \& Davies, 1973) or shared between modalities (Broadbent, 1958; see, e.g., Santangelo, Belardinelli, \& Spence, 2007; Sinnett, Costa, \& Soto-Faraco, 2006, for more recent claims). If sensory modalities have separate attentional capacities, then the level of visual perceptual load will not affect auditory attention and, hence, the incidence of inattentional deafness; on the other hand, if attentional capacity is shared by all modalities, then high visual perceptual load will diminish this resource, leading to reduced processing of auditory stimuli and resulting in a higher incidence of inattentional deafness, as compared to a task with low visual perceptual load.

\section{General method}

Participants performed the same visual discrimination task used by Cartwright-Finch and Lavie (2007). A central cross shape was briefly presented $(150 \mathrm{~ms})$ in each trial. One of the cross arms was green, and the other was blue, and one was slightly longer than the other. In the low-load condition, participants were asked to indicate which arm was blue, and in the high-load condition, they were asked to indicate which of the arms was longer. The subtle line length discrimination task in the high-load condition should demand considerably more attentional resources than the low-load color detection task
(Bonnel, Possamaï, \& Schmitt, 1987; Lavie, 1995), and such a manipulation of load has been shown to reduce both the influence of distractors on task reaction times (RTs) and rates of reports of awareness in previous visual perceptual load studies (for reviews, see Lavie, 2005, 2010). In the seventh trial, a critical stimulus (CS) was presented-here, a taskunrelated pure tone. Awareness of the tone was assessed immediately following the task response. A control trial followed this critical trial, in which the same tone was presented, but this time the participants were instructed to ignore the cross stimulus and to just listen for the tone. Any participant who failed to notice the tone in this control trial was excluded from the analysis.

\section{Experiment 1}

\section{Method}

Participants Fifty six participants were recruited at University College London (UCL). Of these, 7 were excluded and replaced, either because they did not report awareness of the CS in the control trial, or because they responded incorrectly to the cross task in the critical trial, or because their mean error rate on the cross task was greater than $40 \%$. The mean age of those included was 22.0 years $(S D=$ 4.8 years), and there were 31 males. All of the participants in this experiment, and in the rest of the experiments reported, had normal or corrected-to-normal vision, normal hearing, and were naive to the purposes of the experiment.

Apparatus and stimuli The experiments were created and run with E-Prime 1.2 (Psychology Software Tools, Inc., Sharpsburg, PA) on a Dell PC attached to a 15-in. monitor. Auditory stimuli were played to the participants through a pair of Philips SBC HP160 stereo headphones. A viewing distance of $57 \mathrm{~cm}$ was maintained throughout the experiment. In each trial, a cross stimulus was presented (see Fig. 1 for an example display), centered at fixation, of which one arm was green (RGB values: 0, 204, 0) and the other, blue (RGB values: 0, 183, 255), whereas the intersection of the arms was black (RGB values: $0,0,0$ ). One arm was slightly longer than the other $\left(3.6^{\circ}\right.$ and $\left.3.8^{\circ}\right)$. Each arm was equally likely to be blue or green and long or short, but one arm was always blue and the other green, and one was always long and one short. The cross was displayed inside a gray rectangle (RGB values: 204, 204, 204) subtending $6^{\circ} \times 5^{\circ}$ with a black background. At all other times, the display background was black.

White noise at $48 \mathrm{~dB}$ was played continuously for $1.9 \mathrm{~s}$ during each trial, starting at the beginning of each trial and stopping $2 \mathrm{~s}$ before the end. In the critical and control trials, a $180-\mathrm{Hz}$ pure tone at $28 \mathrm{~dB}$ of either 100 or $150 \mathrm{~ms}$ in 
Fig. 1 (Top panel) Each block contained eight trials. (Left panel) In the first six trials, a 900-ms fixation dot was followed by the cross stimulus for $150 \mathrm{~ms}$, and subsequently by a blank screen for 2,850 ms, during which time participants made their cross task response. White noise was played at $48 \mathrm{~dB}$ over headphones for $1,900 \mathrm{~ms}$ from the start of each trial, ending $850 \mathrm{~ms}$ into the response screen. (Right panel) In the seventh and eighth trials, the critical stimulus (a 180-Hz, 28-dB pure tone) was presented concurrently with the cross stimulus for either 100 or $150 \mathrm{~ms}$, embedded in the white noise. Awareness of the critical stimulus was assessed immediately following the task response with an on-screen prompt
Block

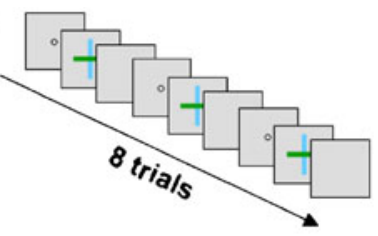

Trials 1-6

Trials 7-8 (critical and control trials)

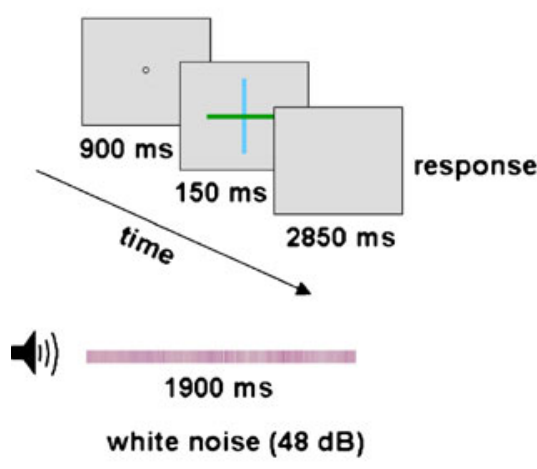

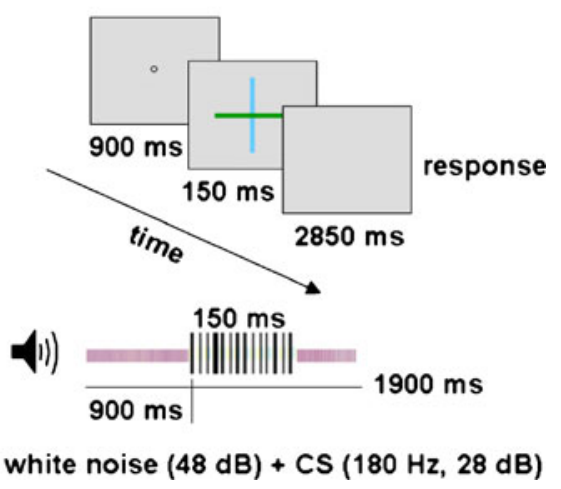

duration (equally likely) was presented at the onset of the cross. All auditory stimuli were prepared with $\mathrm{NCH}$ Tone Generator 2.01 (NCH Software, Inc., Greenwood Village, $\mathrm{CO}$ ). Sound pressure levels were measured in $\mathrm{dB}$ using a Ruel \& Kjær Precision 2209 sound meter at the earpiece of the headphones in a soundproofed room.

The stimuli were presented in one block of eight trials, with the CS presented in the final two trials (the critical and control trials). One of a set of four different stimulus displays (two colors $\times$ two lengths) was randomly selected with equal probability for each trial.

Procedure See Fig. 1 for a graphical illustration of the experimental procedure. A white fixation dot was presented at the center of a black screen for $900 \mathrm{~ms}$ at the start of each trial, followed by the cross display for $150 \mathrm{~ms}$, and subsequently a black screen for $2.85 \mathrm{~s}$, during which participants could make the cross task response. This time window elapsed regardless of whether or not a response was made. The experimenter pressed the space bar to start the next trial. In the low-load condition, the participants' task was to decide which arm of the cross was blue, whereas in the high-load condition, their task was to decide which arm was longest. Participants were instructed to make their cross response as accurately as possible, and responses were not speeded. The " $\mathrm{K}$ " key was pressed if the horizontal arm of the cross was blue or longer, and the "L" key was pressed if it was the vertical arm. There was no feedback for cross task responses except during the practice trials. Participants were informed that they should wear headphones for the duration of the experiment and that a "hiss" (white noise) would be played during each trial in order to aid concentration by blocking out noise from people passing the testing room. Participants seemed to take this instruction at face value and did not seem suspicious. Awareness of the CS in the critical trial was assessed with an on-screen prompt, immediately following the cross task response, asking whether the participant had noticed anything different about the sound coming through the headphones during the last trial. They were subsequently asked to describe to the experimenter what they had heard (even if they said they had not noticed anything different). In the final trial, participants were instructed to ignore the cross and to just listen carefully to the sound coming through the headphones. They were then asked to describe what they had heard.

Each participant performed either low- or high-load trials (selected alternately; low for half of the participants, high for the other half). Before starting the experiment, each participant was shown six example trials, the first three with a cross duration of $1,050 \mathrm{~ms}$ to enable clearer viewing of the stimulus, and the next three at the normal experimental duration (150 ms). Feedback was given on screen after each of these practice trials. Each participant then completed a single experimental block of eight trials, of which the seventh was the critical trial and the eighth was the control trial, during both of which the CS was presented. Any participant who failed to report the presence of the CS in the control trial or who responded incorrectly to the cross task in the critical trial was excluded from the analysis and replaced with a new participant.

Results and discussion

Cross task A one-way ANOVA on mean error rates in the low- and high-load conditions revealed that error rates were 
significantly higher in the high-load condition $(M=14 \%)$ than in the low-load condition $(M=0 \%), F(1,54)=24.83$, $M S E=2,457.88, p<.001, \eta_{\mathrm{p}}{ }^{2}=.315$ (two tailed, as is every statistical test in this article). Our manipulation of visual perceptual load was therefore effective.

CS awareness Participants were considered to have been aware of the CS if they reported hearing something different in the critical trial. As is shown in Fig. 2, there was a clear effect of visual perceptual load on inattentional deafness, with 21 out of 28 reporting awareness in the lowload condition, and only 7 of 28 reporting awareness of the CS in the high-load condition, $\chi^{2}(1, N=56)=14.00$, $p<.001$. The duration of the CS (either 100 or $150 \mathrm{~ms}$ ) had no effect on the modulation of inattentional deafness by load: 11 out of 16 participants reported awareness of the 100-ms CS in the low-load condition, whereas 4 out of 16 did in the high-load condition, $\chi^{2}(1, N=32)=6.10$, $p<.05$; likewise, 10 out of 12 reported awareness of the $150-\mathrm{ms} \mathrm{CS}$ in the low-load condition, whereas 3 out of $12 \mathrm{did}$ so in the high-load condition, $\chi^{2}(1, N=24)=$ $8.20, p<.01$. Thus, high perceptual load in a visual attention task resulted in fewer incidences of awareness of a task-unrelated auditory stimulus. These results provide preliminary support for the notion of a shared perceptual processing resource between the auditory and visual modalities.

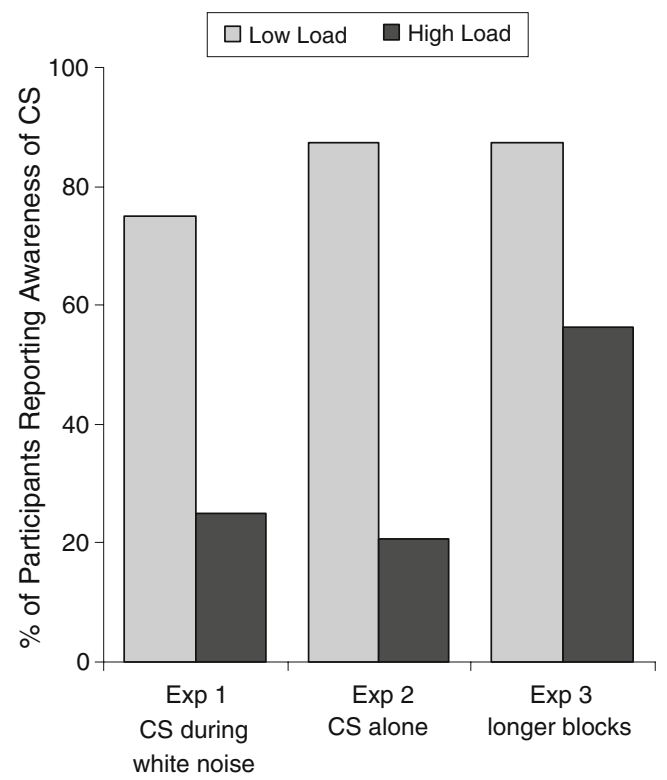

Fig. 2 Percentages of participants reporting awareness of the critical stimulus in the low- and high-load conditions in Experiment $1(N=56)$, in which the stimulus was embedded in $1.9 \mathrm{~s}$ of white noise that was presented on every trial; Experiment $2(N=48)$, in which the stimulus was presented alone; and Experiment $3(N=64)$, which featured longer blocks of trials

\section{Experiment 2}

In Experiment 1, we demonstrated that perceptual load in a visual attention task modulated participants' awareness of a task-unrelated pure tone embedded in white noise. In Experiment 2, we sought to examine whether the load on visual attention would reduce awareness of the same sound (i.e., of equal pitch, volume, and duration) when it was presented alone, unmasked by the white noise, thereby exhibiting a higher signal-to-noise ratio. In addition, the presentation of white noise in all experimental trials in Experiment 1 might have led participants to treat all sound as a distraction and to attempt to actively ignore it. In Experiment 2, we therefore removed the presentation of white noise from all trials. A pure tone of the same volume as in Experiment 1 was presented in the critical trial with no accompanying white noise. It was now, therefore, the first sound that we presented to each participant. A more frequent failure to notice this sound in the highperceptual-load condition of Experiment 2 would confirm an effect of load on inattentional deafness rather than an effect on the active ignoring of distractors.

\section{Method}

Participants Forty-eight new participants were recruited at UCL. Nine of the participants were excluded and replaced, either because they did not report awareness of the CS in the control trial, or because they responded incorrectly to the cross task in the critical trial, or because their mean error rate on the cross task was greater than $40 \%$. The mean age of those included was 21.1 years $(S D=3.1$ years), and there were 19 males. All of the participants in this experiment had normal or corrected-to-normal vision, normal hearing, and were naïve to the purposes of the experiment.

Stimuli and procedure The apparatus, stimuli, and procedure were the same as in Experiment 1, except that no white noise was played through the headphones on each trial. The same $180-\mathrm{Hz}$ pure tone at $28 \mathrm{~dB}$ of either 100 or $150 \mathrm{~ms}$ duration (equally likely) was presented at the onset of the cross. Participants were informed that they should wear headphones for the duration of the experiment in order to aid concentration by blocking out noise from people passing the testing room. Participants seemed to take this instruction at face value and did not appear to be suspicious.

\section{Results and discussion}

Cross task The mean error rate was again significantly higher in the high-load condition $(M=17 \%)$ than in the low-load condition $(M=3 \%), F(1,46)=11.62, M S E=$ 
$2,241.33, p=.001, \eta_{\mathrm{p}}{ }^{2}=.202$, confirming that our manipulation of visual perceptual load was effective.

CS awareness Visual perceptual load strongly influenced the incidence of inattentional deafness (see Fig. 2), with 21 out of 24 reporting awareness of the CS in the low-load condition and only 5 out of 24 reporting it in the high-load condition, $\chi^{2}(1, N=48)=21.48, p<.001$. As with Experiment 1, the duration of the CS (either 100 or $150 \mathrm{~ms}$ ) had no effect on the modulation of inattentional deafness by visual perceptual load: 10 out of 12 participants reported awareness of the 100-ms CS in the low-load condition, whereas 2 out of 12 did in the high-load condition, $\chi^{2}(1, N=24)=10.70, p<.01$; likewise, 11 out of 12 reported awareness of the 150 -ms CS in the low-load condition, whereas 3 out of 12 did in the high-load condition, $\chi^{2}(1, N=24)=11.00, p<.001$. Experiment 2 therefore demonstrated that high perceptual load in a visual attention task reduces auditory awareness, thereby producing inattentional deafness, even with an unmasked tone and when people are not actively ignoring sound.

\section{Experiment 3}

We account for the effect of visual task load on auditory awareness by suggesting that visual perceptual processing consumed more attentional capacity in the high-load task than in the low-load task, leaving less capacity available to process task-irrelevant stimuli. However, because the participants in Experiments 1 and 2 performed exclusively either a low- or a high-load task, alternative accounts of the results in terms of differences in the overall level of motivation, vigilance, task engagement, or performance strategies remained viable. For example, upon receiving the instructions and encountering the first few example trials, those who performed the high-load task might have decided to engage more in the task than those who performed the low-load task. The effect of load on awareness would then be attributable to participants' intentions rather than to the availability of perceptual processing capacity. To rule out these alternative accounts, in Experiment 3 we randomly intermixed low- and high-visual-load tasks within a longer block of 143 trials. In addition, we changed the low-load cross task from a color discrimination to a line length discrimination with a far greater line length difference than in the high-load condition, so that the same task was performed in all trials. Since participants would know that the task involved both levels of load and would not know the level of load on each trial in advance, alternative accounts of the effects of load in terms of intention and overall task engagement strategies could be ruled out. In addition, participants' overall levels of vigilance and motivation would be constant across the randomly intermixed load trials, and so could not account for any effect of load on awareness.

\section{Method}

Participants Sixty-four new participants were recruited at UCL. Of these, 25 participants were excluded and replaced, either because they did not report awareness of the CS in the control trial, or because they responded incorrectly to the cross task in the critical trial, or because their mean error rate on the high-load cross task was greater than $40 \%$. The mean age of those included was 22.2 years $(S D=$ 3.7 years), and there were 19 males. All of the participants in this experiment had normal or corrected-to-normal vision, normal hearing, and were naïve to the purposes of the experiment.

Stimuli and procedure For the high-perceptual-load condition, the same stimuli were used as in Experiments 1 and 2. For the low-load condition, the task was now the same as for the high-load condition, but the stimuli were now different: the short arm was considerably shorter $\left(1.4^{\circ}\right)$ than the long arm $\left(3.8^{\circ}\right)$. The colors of the cross arms were unchanged but were of no consequence. Responses were now speeded, and the 2.85-s blank screen following cross offset terminated on response. Feedback was given following each trial, in the form of the word "correct" in blue or "incorrect" in red, at the center of the screen for $500 \mathrm{~ms}$. There was no feedback given in the critical trial. The CS was the same as in Experiments 1 and 2, except that it was always of 150-ms duration, and, as in Experiment 2, no white noise was played.

The stimuli were presented in two blocks of 72 trials with the CS presented in the final trial (the critical trial), and the control trial was performed afterward. One of a set of eight different stimulus displays (two colors $\times$ four lengths) was randomly selected with equal probability for each trial. The exception to this was the critical trial, for which load was counterbalanced across participants. The practice block now consisted of 18 trials, also containing randomly intermixed low- and high-load trials. In all other respects, the stimuli and apparatus were the same as those for Experiment 2.

\section{Results and discussion}

Cross task The greater number of trials in this experiment allowed us to analyze RTs. Trials in which the search response was incorrect and those in which the RT was greater than $1.5 \mathrm{~s}$ were excluded from the RT analysis. This resulted in excluding $14.6 \%$ trials from the analysis. A one-way ANOVA on mean RTs and error rates in the 
low- and high-perceptual-load conditions revealed that mean RTs were significantly longer in the high-load condition $(M=949 \mathrm{~ms})$ than in the low-load condition ( $M$ $=649 \mathrm{~ms}), F(1,63)=424.59, M S E=6,749.45, p<.001$, $\eta_{\mathrm{p}}{ }^{2}=.871$, and error rates in the high-load condition $(M=$ $19 \%)$ were significantly higher than in the low-load condition $(M=2 \%), F(1,63)=273.83, M S E=34.21, p$ $<.001, \eta_{\mathrm{p}}{ }^{2}=.813$. Our manipulation of load in this experiment was therefore as effective as in the blocked design we employed in the previous experiments.

CS awareness Just as with the previous experiments, there was a clear effect of visual perceptual load on awareness of the CS (see Fig. 2), with 28 out of 32 reporting awareness in the low-load condition and 18 of 32 reporting awareness in the high-load condition, $\chi^{2}(1, N=64)=7.70, p=.005$.

Since we replicated the effect of visual perceptual load found in Experiments 1 and 2, but this time with participants performing both low- and high-load trials, randomly intermixed within blocks, and with the same task for both conditions of load, interpretations of the results in terms of motivation, vigilance, task engagement, or strategy can be ruled out.

Although the effect of load on awareness was robust (a reduction of 31\%), the effect was stronger in Experiments 1 and 2 (reductions of $50 \%$ and $67 \%$, respectively). This difference could reflect improvement in the high-load task over the much longer series of trials (143 instead of 6). Alternatively, it could indicate that there was a vigilance or strategy component to the effect in Experiments 1 and 2 that was abolished when trials were randomly intermixed within blocks.

\section{General discussion}

The present research establishes a new equivalent of inattentional blindness in the auditory domain, namely "inattentional deafness," and highlights the level of perceptual load in a visual task as a critical determinant of this effect. In three experiments, our results consistently showed that people fail to notice the mere presence of a simple auditory tone presented unexpectedly on the last trial while performing a crosshair line judgment task (concerning the line length or color). Importantly, the level of perceptual load in this visual discrimination task modulated the incidence of inattentional deafness. The high-load task, which required a subtle line length discrimination, led to a far greater rate of failing to notice the sound as compared to the low-load task, which required a very obvious line length or line color judgment. Importantly, this effect remained when load conditions were randomly intermixed within a longer series of trials. Thus, inattentional deafness in our study was clearly influenced by the level of visual perceptual load in the task rather than by any differences in motivation, vigilance, task engagement, or strategy (cf. Theeuwes, Kramer, \& Belopolsky, 2004).

These findings establish the phenomenon of inattentional deafness under visual load and extend the load theory of attention and cognitive control to address the cross-modal effect of visual attentional load on awareness of auditory information. They suggest that the elementary process of noticing the mere presence of a sound depends on an attentional capacity resource that is shared between the modalities of vision and hearing. This conclusion makes a novel contribution to the understanding of the determinants of awareness failures, and to capacity limits across the different senses, and it has important implications with regard to everyday tasks such as driving. We discuss these contributions in the following sections.

Failures of awareness within and between sensory modalities

A large body of research has established inattentional blindness in the visual domain and highlighted the critical role of perceptual load. This research has revealed that people may fail to notice various unattended stimuli-for example, simple shapes, meaningful objects, words or events (Most, Simons, et al., 2001; Neisser \& Becklen, 1975; Rock \& Gutman, 1981; Simons \& Chabris, 1999)while attending to a task involving high perceptual load (e. g., keeping a separate count of different types of ball passes). The effects of inattentional blindness have generalized across measures of high-level recognition-for example, identifying words or comprehending the content of an ignored passage of text (Mack \& Rock, 1998; Moray, 1959; Neisser, 1968; Rees, Russell, Frith, \& Driver, 1999) - and measures of lower-level visual detection — such as noticing the mere presence of a simple shape (Mack \& Rock, 1998; Most, Scholl, Clifford, \& Simons, 2005; Most, Simons, et al., 2001).

The effects of inattentional blindness have also generalized across both long and short delays between the presentation of the critical stimulus and the measure of awareness: for example, when awareness reports are collected at the end of a presentation video or stream (e.g., Most, Schroll, et al., 2005; Most, Simons et al., 2001; Neisser \& Becklen, 1975; Rock \& Gutman, 1981; Simons \& Chabris, 1999), or when these are collected shortly after presentation of the critical stimulus in the final experiment trial (e.g., Cartwright-Finch \& Lavie, 2007; Downing, Bray, Rogers, \& Childs, 2004; Mack \& Rock, 1998). Therefore, memory failure, which may contribute to inattentional blindness in paradigms featuring a long delay before awareness reports are made or 
involving the full semantic content of words or a passage of text, is not a likely alternative explanation for the phenomenon of inattentional blindness in general. The interpretation of inattentional blindness as reflecting a perceptual detection failure has received support from a recent study that demonstrated that high attentional load reduces the perceptual sensitivity of visual, task-irrelevant stimuli and has no effect on response criterion, in a paradigm in which awareness reports were made as soon as the critical stimulus had been presented, or even before the task response (Macdonald \& Lavie, 2008).

In contrast, inattentional deafness has so far only been demonstrated in studies involving the rather high-level process of semantic word recognition, in which awareness reports followed a fairly long delay at the end of a presentation stream. For instance, Moray (1959) demonstrated that at the end of the experiment, following performance of a dichotic listening paradigm, people failed to recognize the content of words presented in the unattended ear, even if these had been repeated many times during the experiment.

Sinnett et al. (2006), in a more recent and rather thorough investigation of the effects of attention on word recognition within and between sensory modalities, established that people fail to recognize words presented auditorily while they attend to a rapid stream of either pictures or sounds. Recognition memory for these words was considerably lower for both within- and betweenmodality and conditions than when the words were attended in a control condition. These findings clearly show that word recognition depends on a capacity-limited resource common to vision and hearing. However, the retrospective measure of recognition, following a stream of words, leaves open the alternative explanation that the words had been forgotten rather than not perceived. Furthermore, the comparison of attended and unattended words in different blocks could have involved intentions or strategy, in addition to any effect of perceptual load.

Our findings, however, support the authors' attentional interpretation, while ruling out strategy-based alternative accounts of the effect of load (see Exp.3). Importantly, our findings extend previous inattentional blindness research in demonstrating that perceptual load in a visual task modulates the fundamental process of noticing the presence of a simple sound.

The short stimulus and response durations used in our paradigm rendered an account of inattentional deafness in terms of forgetting the sound had occurred rather than not perceiving it unlikely. Nevertheless, it is possible that some instances of inattentional deafness in our task involved a failure to encode the sound into memory or to consolidate a weak memory trace over the short delay incurred by preparing and making a task response, rather than a failure to perceive the sound in the first place (see, e.g., Moore,
2001). In addition, the present results are limited to the case of unexpected sounds. A future study adopting Macdonald and Lavie's (2008) paradigm to test detection sensitivity for expected auditory stimuli could address both of these issues. The present findings, however, do not hinge on these factors. Irrespective of any potential contribution of failures to encode or consolidate a memory trace of the sound, the present findings clearly demonstrate that the subjective experience of noticing a sound depends on the level of visual perceptual load in the task being undertaken. This is not only important for the theoretical understanding of the determinants of awareness but is also highly applicable to real-world settings: It is often the case that important auditory information is unexpected (e.g., the sound of your car alarm), and the potential applied benefits of understanding the task determinants of the likelihood of failures to notice such auditory information remain, irrespective of whether the failures to notice are purely perceptual or involve a failure to encode into memory.

Further future research could generalize the effect of load on the awareness of a sound that we report here across different manipulations of perceptual load in a visual task; that is, the stimuli could be varied-for example, the number of nontargets or similarity of nontargets to targets in a search task-rather than the task (e.g., Lavie, 1995; Lavie \& Cox, 1997; Macdonald \& Lavie, 2008). In addition, it will be important to establish whether the effects we report here can be distinguished from the effects of visual task difficulty. Lavie and de Fockert (2003) demonstrated that under certain conditions the effects of visual perceptual load can be distinguished from the effects of extreme sensory degradation and an overall increase in task difficulty. When participants performed a letter identification task, extreme degradation of the sensory input that rendered the target letter stimulus barely visible subjected task performance to sensory "data limits" rather than attentional resource limits (see Norman \& Bobrow, 1975), and therefore did not reduce distractor processing. Although the high-load line length discrimination task we used was demanding, the length difference was clearly visible, given that accuracy rates were well above chance $(86 \%, 83 \%$, and $81 \%$ in the three experiments, respectively). Hence, the task demands were in the range of resource limits rather than data limits, and therefore any effects of the task reflect attentional resource load rather than sensory degradation. Future research could go further, however, to distinguish the effect of perceptual load from that of sensory degradation by using peri-threshold discrimination tasks in the high-load condition and assess the effect on the incidence of inattentional blindness or deafness.

The effect we have established involved a pure tone and generalized across two different signal-to-noise ratios, since in Experiment 1 the pure tone was accompanied by white noise, producing a sound of low signal-to-noise ratio, and 
in Experiment 2 it was not, thereby rendering the pure tone of a higher signal-to-noise ratio. Future research should also address whether the effect found here generalizes to sounds varied across a range of dimensions - for example, volume, pitch, and timbre-while also considering the signal-tonoise ratio.

\section{Capacity limits across vision and hearing}

There is a growing body of evidence for a shared attentional capacity between the modalities of vision and hearing. For example, the aforementioned study by Sinnett et al. (2006) showed reduced word recognition rates when attention was paid to a demanding stimulus stream, irrespective of whether the words and stream were presented in the same or different modalities. Furthermore, a few recent studies have demonstrated that the cost to performance involved in coordinating two tasks, as compared to a single task, is not reduced-and in fact in some cases is increased-when the two tasks draw on separate sensory modalities (e.g., vision and hearing) rather than the same sensory modality (see, e.g., BrandD’Abrescia \& Lavie, 2008; Hunt \& Kingstone, 2004; Jolicœur, 1999). In one other example, Santangelo et al. (2007), using a peripheral cuing paradigm, showed that focusing attention on a central stream of visual or auditory characters involving a high-perceptual-load task (letter or digit identification) led to reduced cuing effects for peripheral cues, irrespective of whether the cues were presented in the same sensory modality as the central stream or a different one.

The conclusion from our study, that the process of noticing a simple tone depends on a shared attentional capacity between hearing and vision, is consistent with these previous studies. Indeed, it offers an explanation for some of these previous findings in terms of reduced awareness of the cue stimulus (in Santangelo et al., 2007) or of the ignored words (in Sinnett et al., 2006) under their task conditions of high perceptual load.

The discrepancy between these demonstrations of shared capacity between vision and hearing and other lines of work that have suggested that some attentional capacities appear to be modality specific (e.g., Duncan et al., 1997; SotoFaraco, Morein-Zamir, \& Kingstone, 2005; Tellinghuisen \& Nowak, 2003; Treisman \& Davies, 1973) may in some cases be accounted for by task-specific effects. For example, in one previous study, high visual perceptual load was found to reduce response competition effects from visual distractor letters, but the same manipulation of visual perceptual load had no effect on response competition effects from auditory distractor letters (Tellinghuisen \& Nowak, 2003). However, in this study, the presentation durations of the visual and auditory distractor letters were different: Whereas the visual distractor letters were presented for the same duration as the target letters, the auditory distractor letters were presented for a longer duration, ending $200 \mathrm{~ms}$ after the offset of the visual target displays. As such, there was a greater temporal overlap between the processing of the visual target and the auditory distractor in the high-load condition (in which the target search took longer) than in the low-load condition, and this may have offset the effect of load.

It is, of course, possible that some processing capacities are modality specific, while others draw on a shared crossmodal resource. For example, previous findings that perception of visual motion is reduced by attending to a high-load visual stimulus stream but not to an auditory word stream (Rees, Frith, \& Lavie, 1997, 2001) may indicate that the perception of visual motion only suffers from visual capacity limits. In addition, the attentional blink - the reduction in perception of a second target when it is presented within $300 \mathrm{~ms}$ of the first target in an RSVP stream-appears to have modality-specific limits: It is only found when the two targets are presented within the same modality (e.g., Duncan et al., 1997; Soto-Faraco \& Spence, 2002). This may indicate that the temporal limits on perceptual processing are modality specific, perhaps due to the different timings of the information processing in each modality.

While our study does not attempt to resolve the discrepancy between studies indicating shared or separate capacity limits between the senses, it does make clear that in the case of awareness of the mere presence of a simple tone, vision and hearing share a common processing resource, rather than each having its own pool. Indeed, the sizes of the effects of visual perceptual load on incidences of inattentional deafness that we report are of magnitudes similar to those reported previously for the same manipulation of visual perceptual load on incidences of inattentional blindness. In our experiments, high load increased incidences of inattentional deafness by $49 \%$ on average, whereas Cartwright-Finch and Lavie (2007) found that the same manipulation of load increased incidences of inattentional blindness by $44 \%$ on average (Exps. 1 and 3). Therefore, it appears that, at least for measures of awareness, the visual and auditory modalities share the same capacity-limited resource.

Finally, our demonstration that visual perceptual task load leads to the failure to notice the presence of a taskunrelated auditory stimulus has significant implications for everyday life. For instance, our results imply that people would be less likely to notice an auditory alarm while engaged in a high-visual-load computer task, or more importantly, the sound of a car horn while attending to a visually loaded billboard. The implications of research on inattentional blindness for daily life tasks such as driving have begun to be noted by some public authorities-for example, Traffic for London, which has begun a campaign to alert drivers to the dangers of inattentional blindness. 
The present research suggests that they also ought to consider the effects of inattentional deafness under load.

Author Note This research was supported by a Wellcome trust grant to N.L. We thank Aura Rico and Meiqi Ang for help in running the experiments reported.

Open Access This article is distributed under the terms of the Creative Commons Attribution Noncommercial License which permits any noncommercial use, distribution, and reproduction in any medium, provided the original author(s) and source are credited.

\section{References}

Allport, D. A., Antonis, B., \& Reynolds, P. (1972). On the division of attention: A disproof of the single channel hypothesis. The Quarterly Journal of Experimental Psychology, 24, 225-235.

Bonnel, A.-M., Possamaï, C.-A., \& Schmitt, M. (1987). Early modulation of visual input: A study of attentional strategies. The Quarterly Journal of Experimental Psychology, 39A, 757776.

Brand-D'Abrescia, M., \& Lavie, N. (2008). Task coordination between and within sensory modalities: Effects on distraction. Perception \& Psychophysics, 70, 508-515.

Broadbent, D. E. (1958). Perception and communication. New York: Academic Press.

Carmel, D., Saker, P., Rees, G., \& Lavie, N. (2007). Perceptual load modulates conscious flicker perception. Journal of Vision, 7(14), 14:1-13. doi:10.1167/7.14.14

Cartwright-Finch, U., \& Lavie, N. (2007). The role of perceptual load in inattentional blindness. Cognition, 102, 321-340. doi:10.1016/ j.cognition.2006.01.002

Downing, P. E., Bray, D., Rogers, J., \& Childs, C. (2004). Bodies capture attention when nothing is expected. Cognition, 93, B27B38. doi:10.1016/j.cognition.2003.10.010

Duncan, J., Martens, S., \& Ward, R. (1997). Restricted attentional capacity within but not between sensory modalities. Nature, 387, 808-810. doi:10.1038/42947

Hunt, A. R., \& Kingstone, A. (2004). Multisensory executive functioning. Brain and Cognition, 55, 325-327. doi:10.1016/j. bandc.2004.02.072

Jenkins, R., Lavie, N., \& Driver, J. (2005). Recognition memory for distractor faces depends on attentional load at exposure. Psychonomic Bulletin \& Review, 12, 314-320.

Jolicœur, P. (1999). Dual-task interference and visual encoding. Journal of Experimental Psychology. Human Perception and Performance, 25, 596-616.

Lavie, N. (1995). Perceptual load as a necessary condition for selective attention. Journal of Experimental Psychology. Human Perception and Performance, 21, 451-468.

Lavie, N. (2005). Distracted and confused? Selective attention under load. Trends in Cognitive Sciences, 9, 75-82. doi:10.1016/j. tics.2004.12.004

Lavie, N. (2010). Attention, distraction, and cognitive control under load. Current Directions in Psychological Science, 19, 143-148. doi:10.1177/0963721410370295

Lavie, N., \& Cox, S. (1997). On the efficiency of visual selective attention: Efficient visual search leads to inefficient distractor rejection. Psychological Science, 8, 395-398. doi:10.1111/ j.1467-9280.1997.tb00432.x

Lavie, N., \& de Fockert, J. W. (2003). Contrasting effects of sensory limits and capacity limits in visual selective attention. Perception \& Psychophysics, 65, 202-212.
Lavie, N., Lin, Z., Zokaei, N., \& Thoma, V. (2009). The role of perceptual load in object recognition. Journal of Experimental Psychology. Human Perception and Performance, 35, 13461358. doi:10.1037/a0016454

Lavie, N., \& Tsal, Y. (1994). Perceptual load as a major determinant of the locus of selection in visual attention. Perception \& Psychophysics, 56, 183-197.

Macdonald, J. S. P., \& Lavie, N. (2008). Load induced blindness. Journal of Experimental Psychology. Human Perception and Performance, 34, 1078-1091. doi:10.1037/0096-1523.34.5.1078

Mack, A., \& Rock, I. (1998). Inattentional blindness. Cambridge: MIT Press.

McLeod, P. (1977). A dual task response modality effect: Support for multiprocessor models of attention. The Quarterly Journal of Experimental Psychology, 29, 651-667.

Moore, C. M. (2001). Inattentional blindness: Perception or memory and what does it matter? Psyche: An Interdisciplinary Journal of Research on Consciousness, 7(2).

Moray, N. (1959). Attention in dichotic listening: Affective cues and the influence of instructions. The Quarterly Journal of Experimental Psychology, 11, 56-60.

Most, S. B., Scholl, B. J., Clifford, E. R., \& Simons, D. J. (2005). What you see is what you set: Sustained inattentional blindness and the capture of awareness. Psychological Review, 112, 217 242. doi:10.1037/0033-295X.112.1.217

Most, S. B., Simons, D. J., Scholl, B. J., Jimenez, R., Clifford, E. R., \& Chabris, C. F. (2001). How not to be seen: The contribution of similarity and selective ignoring to sustained inattentional blindness. Psychological Science, 12, 9-17.

Neisser, U. (1968). The processes of vision: Light enables us to see, but optical images on the retina are only the starting point of the complex activities of visual perception and visual memory. Scientific American, 219, 204-214.

Neisser, U., \& Becklen, R. (1975). Selective looking: Attending to visually specified events. Cognitive Psychology, 7, 480-494. doi:10.1016/0010-0285(75)90019-5

Norman, D. A., \& Bobrow, D. G. (1975). On data-limited and resource-limited processes. Cognitive Psychology, 7, 44-64. doi:10.1016/0010-0285(75)90004-3

Rees, G., Frith, C. D., \& Lavie, N. (1997). Modulating irrelevant motion perception by varying attentional load in an unrelated task. Science, 278, 1616-1619.

Rees, G., Frith, C. D., \& Lavie, N. (2001). Processing of irrelevant visual motion during performance of an auditory attention task. Neuropsychologia, 39, 937-949.

Rees, G., Russell, C., Frith, C. D., \& Driver, J. (1999). Inattentional blindness versus inattentional amnesia for fixated but ignored words. Science, 286, 2504-2507.

Rock, I., \& Gutman, D. (1981). The effect of inattention on form perception. Journal of Experimental Psychology. Human Perception and Performance, 7, 275-285.

Santangelo, V., Belardinelli, M. O., \& Spence, C. (2007). The suppression of reflexive visual and auditory orienting when attention is otherwise engaged. Journal of Experimental Psychology. Human Perception and Performance, 33, 137148. doi:10.1037/0096-1523.33.1.137

Simons, D. J., \& Chabris, C. F. (1999). Gorillas in our midst: Sustained inattentional blindness for dynamic events. Perception, 28, 1059-1074. doi:10.1068/p2952

Sinnett, S., Costa, A., \& Soto-Faraco, S. (2006). Manipulating inattentional blindness within and across sensory modalities. The Quarterly Journal of Experimental Psychology, 59, 14251442. doi:10.1080/17470210500298948

Soto-Faraco, S., Morein-Zamir, S., \& Kingstone, A. (2005). On audiovisual spatial synergy: The fragility of the phenomenon. Perception \& Psychophysics, 67, 444-457. 
Soto-Faraco, S., \& Spence, C. (2002). Modality-specific auditory and visual temporal processing deficits. The Quarterly Journal of Experimental Psychology, 55A, 23-40.

Tellinghuisen, D. J., \& Nowak, E. J. (2003). The inability to ignore auditory distractors as a function of visual task perceptual load. Perception \& Psychophysics, 65, 817-828.
Theeuwes, J., Kramer, A. F., \& Belopolsky, A. V. (2004). Attentional set interacts with perceptual load in visual search. Psychonomic Bulletin \& Review, 11, 697-702.

Treisman, A. M., \& Davies, A. (1973). Divided attention to ear and eye. In S. Kornblum (Ed.), Attention and performance IV (pp. 101117). New York: Academic Press. 